4.1 Remarks. Suppose that the hypothesis of a theorem involves convergence $(\sigma)$. It may happen (and does indeed in the case of all theorems in [S]) that convergence $\left(\sigma_{1}\right)$ may be substituted in the hypothesis as follows: each $\sigma$-region which occurs in the proof is a $\sigma_{n}$-region for some $n$; if there is a largest such $n$ call it $N$. Then convergence $\left(\sigma_{N}\right)$ may be substituted in the hypothesis and may, by Theorem I, be replaced by convergence $\left(\sigma_{1}\right)$. Lemma 10 is an example of an exception to this statement; there is no largest $n$ and convergence $(\sigma)$ is essential.

If a series is convergent $\left(\sigma_{1}\right)$ then, for a given $n$, the series is convergent $\left(\sigma_{n}\right)$. Hence, given $\epsilon>0$ there are indices $(p, q)$ such that $\left|A-\sum(R)\right|<\epsilon$ for every $\sigma_{n}$-region $R$ which is a region $(p, q)$. In general $(p, q)$ will depend on $n$; if the choice does not depend on $n$ then the series is convergent $(\sigma)$.

BROWN UNIVERSITY

\title{
THE DIFFERENTIABILITY AND UNIQUENESS OF CON- TINUOUS SOLUTIONS OF ADDITION FORMULAS
}

\section{NELSON DUNFORD AND EINAR HILLE}

The problem of representing a one-parameter group of operators (that is, a family $T_{\xi},-\infty<\xi<\infty$, of bounded linear operators on a Banach space which satisfies $T_{\xi+\zeta}=T_{\xi} T_{\zeta}$ ) reduces according to several well known methods of attack to establishing differentiability of the function $T_{\xi}$ at $\xi=0$. The derivative $A x=\lim _{\xi \rightarrow 0} \xi^{-1}\left(T_{\xi}-I\right) x$ exists as a closed operator with domain $D(A)$ dense, providing $T_{\xi}$ is continuous in the strong operator topology (that is, $\lim _{\xi \rightarrow \xi_{0}} T_{\xi} x=T_{\xi_{0}} x, x \in \mathfrak{X}$ ). It is then possible to assign a meaning to $\exp (\xi A)$ in a natural way and so that $T_{\xi}=\exp (\xi A),-\infty<\xi<\infty$. The operator $A$ is bounded if and only if $T_{\xi}$ is continuous in $\xi$ in the uniform operator topology (that is, $\lim _{\xi \rightarrow \xi_{0}}\left|T_{\xi}-T_{\xi_{0}}\right|=0$ ) in which case $A=\lim _{\xi \rightarrow 0} \xi^{-1}\left(T_{\xi}-I\right.$ ) exists in the uniform topology. This implies that $T_{\xi}$ is an entire function of $\xi$; conversely, if $T_{\xi}$ is analytic anywhere, then $A$ is bounded. These considerations extend to the semi-group case in which $T_{\xi+\xi}=T_{\xi} T_{\zeta}$ is known to hold only for positive values of the parameters, although 1947.

Presented to the Society, August 14, 1944; received by the editors February 17, 
the number of distinct cases is much larger and, in particular, analyticity does not imply that $A$ is bounded.

It is a matter of natural curiosity to ask whether or not similar results hold if the semi-group law $f(\xi+\zeta)=f(\xi) f(\zeta)$ is replaced by an arbitrary addition formula. The results presented in the following correspond to the case of an analytical group (that is, continuity in the uniform topology). The strong operator topology leads to particular difficulties which have been overcome only in part, but we hope to return to this case on a future occasion.

In this note we consider the differentiability and uniqueness of continuous solutions $f(\xi)$ of the equation

$$
f(\xi+\zeta)=G[f(\xi), f(\zeta)], \quad 0 \leqq \xi, \zeta, \xi+\zeta \leqq \omega,
$$

where $G[\alpha, \beta]$ is a symmetric complex function analytic for $\alpha, \beta$ in the closure of a domain $\Delta$ bounded by a rectifiable Jordan curve. The solutions considered are functions $f(\xi)$ on $0 \leqq \xi \leqq \omega$ to a commutative complex Banach algebra $B$ with unit $e$. We define $G[u, v]$ only for those $u, v \in B(\Delta)$, the subset of $B$ consisting of elements $x$ whose spectrum $\sigma x \subset \Delta$. For such $u, v$ we define $G[u, v]$ by the double resolvent integral

$$
G[u, v]=\frac{1}{(2 \pi i)^{2}} \int_{\mathrm{r}_{u}} \int_{\mathrm{r}_{v}} G[\alpha, \beta] R(\alpha, u) R(\beta, v) d \alpha d \beta,
$$

where $R(\alpha, u)=(\alpha e-u)^{-1}$ and $\Gamma_{u}, \Gamma_{v}$ are oriented envelopes in $\Delta$ of $\sigma u, \sigma v$ respectively. Thus by a solution of (1) is meant a function $f(\xi)$ on $0 \leqq \xi \leqq \omega$ to $B(\Delta)$ which satisfies $(1)$.

THEOREM. If $f(\xi)$ is a continuous $B$-valued solution of (1) and if $G_{1}[f(0), f(0)]$ has an inverse $\left(G_{1}[\alpha, \beta]=(\partial / \partial \alpha) G[\alpha, \beta]\right)$, then $f(\xi)$ has derivatives of all orders and $f^{\prime}(\xi)=G_{1}[f(0), f(\xi)] f^{\prime}(0)$. If $g(\xi)$ is any other continuous solution of $(1)$ with $g(0)=f(0)$ and $g^{\prime}(0)=f^{\prime}(0)$, then $g(\xi) \equiv f(\xi)$. If $\phi(\xi)$ is a nonconstant scalar analytic solution of (1) then the only continuous $B$-valued solution of (1) with $f(0)=\phi(0) e$ is given by $f(\xi)=\phi\left(f^{\prime}(0) \xi\right)$ in a neighborhood $0 \leqq \xi \leqq \rho$.

LEMmA. Let $Q[\alpha, \beta, \gamma]=(G[\alpha, \gamma]-G[\beta, \gamma])(\alpha-\beta)^{-1}, \alpha, \beta, \gamma \in \Delta$. If $f(\xi)$ is continuous on $0 \leqq \xi \leqq \omega$ to $B(\Delta)$ and $0 \leqq \eta, \zeta \leqq \omega$ then uniformly with respect to $\zeta$ we have

$$
\begin{gathered}
\lim _{\xi \rightarrow \eta} Q[f(\xi), f(\eta), f(\zeta)]=G_{1}[f(\eta), f(\zeta)], \\
Q(u, v, w)(u-v)=G(u, w)-G(v, w), \quad u, v, w \in B(\Delta) .
\end{gathered}
$$


First note that since $f(\xi)$ is continuous in $[0, \omega]$ the range of $f(\xi)$ is a closed connected compact set $R \subset B(\Delta)$. If $\Phi=U_{\sigma x}, x \in R$, then $\Phi$ is a closed subset of $\Delta$ and if $\Gamma$ is an oriented envelope of $\Phi$ in $\Delta$, then $R[\alpha, f(\xi)]$ is a continuous function of $(\alpha, \xi)$ for $\alpha \in \Gamma, 0 \leqq \xi \leqq \omega$. There is consequently a finite positive constant $M=M(\Gamma)$ such that

$$
|R[\alpha, f(\xi)]| \leqq M(\Gamma), \quad \alpha \in \Gamma, 0 \leqq \xi \leqq \omega .
$$

Further, $\lim _{\xi \rightarrow \eta} R[\alpha, f(\xi)]=R[\alpha, f(\eta)]$ uniformly with respect to $\alpha$ on $\Gamma$. It follows that uniformly in $\zeta, 0 \leqq \zeta \leqq \omega$,

$$
\begin{aligned}
\lim _{\xi \rightarrow \eta} Q[f(\xi), f(\eta), f(\zeta)] & \\
= & -\frac{1}{8 \pi^{3} i} \int_{\Gamma} \int_{\Gamma} \int_{\Gamma} Q[\alpha, \beta, \gamma] R[\alpha, f(\eta)] R[\beta, f(\eta)] R[\gamma, f(\zeta)] d \alpha d \beta d \gamma \\
= & \frac{1}{8 \pi^{3} i} \int_{\Gamma} \int_{\Gamma} \int_{\Gamma} Q(\alpha, \beta, \gamma) \frac{R[\alpha, f(\eta)]-R[\beta, f(\eta)]}{\alpha-\beta} R[\gamma, f(\zeta)] d \alpha d \beta d \gamma \\
= & \frac{1}{8 \pi^{3} i} \int_{\Gamma_{1}} \int_{\Gamma_{1}} d \beta d \gamma R[\gamma, f(\zeta)]\left\{\int_{\Gamma} \frac{Q(\alpha, \beta, \gamma) R[\alpha, f(\eta)]}{\alpha-\beta} d \alpha\right. \\
& \left.\quad-R[\beta, f(\eta)] \int_{\Gamma} \frac{Q(\alpha, \beta, \gamma)}{\alpha-\beta} d \alpha\right\} .
\end{aligned}
$$

Here $\Gamma, \Gamma_{1}$, are oriented envelopes of $\Phi$ in $\Delta$ and $\Gamma_{1}$ is interior to $\Gamma$. Now

$$
\int_{\Gamma} \frac{Q(\alpha, \beta, \gamma)}{\alpha-\beta} d \alpha=2 \pi i Q(\beta, \beta, \gamma)=2 \pi i G_{1}(\beta, \gamma)
$$

so

$\lim _{\xi \rightarrow \eta} Q[f(\xi), f(\eta), f(\zeta)]$

$$
\begin{aligned}
& =\frac{1}{(2 \pi i)^{2}} \int_{\Gamma_{1}} \int_{\Gamma_{1}} G_{1}(\beta, \gamma) R[\beta, f(\eta)] R[\gamma, f(\zeta)] d \beta d \gamma+U \\
& =G_{1}[f(\eta), f(\zeta)]+U .
\end{aligned}
$$

Here

$$
\begin{aligned}
U & =\frac{1}{8 \pi^{3} i} \int_{\mathrm{r}_{1}} \int_{\mathrm{r}_{1}} d \beta d \gamma R[\gamma, f(\zeta)] \int_{\mathrm{r}} \frac{Q(\alpha, \beta, \gamma)}{\alpha-\beta} R[\alpha, f(\eta)] d \alpha \\
& =\frac{1}{8 \pi^{3} i} \int_{\mathrm{r}_{1}} d \gamma R[\gamma, f(\zeta)] \int_{\mathrm{r}} d \alpha R[\alpha, f(\eta)] \int_{\mathrm{r}_{1}} \frac{Q(\alpha, \beta, \gamma)}{\alpha-\beta} d \beta
\end{aligned}
$$


But for $\alpha \in \Gamma$ the last integral is zero so $U=0$. This completes the proof of (3). Equation (4) may be proved in a fashion analogous to the method used for establishing the multiplicative law in the operational calculus involving functions of one variable.

We now proceed to the proof of the theorem. From the contour integral definition of $G_{1}[f(0), f(\zeta)]$ we see that it is continuous in $\zeta$ and hence

$$
\lim _{\alpha \rightarrow 0} \frac{1}{\alpha} \int_{0}^{\alpha} G_{1}[f(0), f(\zeta)] d \zeta=G_{1}[f(0), f(0)] .
$$

Thus we may fix $\alpha<\omega$ so that the integral on the left has an inverse in $B$. From the lemma we have

$$
\lim _{\xi \rightarrow 0} \frac{1}{\alpha} \int_{0}^{\alpha} Q[f(\xi), f(0), f(\zeta)] d \zeta=\frac{1}{\alpha} \int_{0}^{\alpha} G_{1}[f(0), f(\zeta)] d \zeta
$$

Hence

$$
\begin{aligned}
\lim _{\xi \rightarrow 0}\left\{\frac{1}{\alpha} \int_{0}^{\alpha} Q[f(\xi), f(0), f(\zeta)] d \zeta\right\}^{-1} & \\
= & \left\{\frac{1}{\alpha} \int_{0}^{\alpha} G_{1}[f(0), f(\zeta)] d \zeta\right\}^{-1} .
\end{aligned}
$$

From (4) we have

(7) $[f(\xi)-f(\eta)] Q[f(\xi), f(\eta), f(\zeta)]=G[f(\xi), f(\zeta)]-G[f(\eta), f(\zeta)\rfloor$

whence

$$
\begin{aligned}
& \frac{1}{\xi}[f(\xi)-f(0)] \frac{1}{\alpha} \int_{0}^{\alpha} Q[f(\xi), f(0), f(\zeta)] d \zeta \\
& =\frac{1}{\alpha} \int_{0}^{\alpha} \frac{1}{\xi}\{G[f(\xi), f(\zeta)]-G[f(0), f(\zeta)]\} d \zeta \\
& =\frac{1}{\alpha \xi} \int_{0}^{\alpha}[f(\xi+\zeta)-f(\zeta)] d \zeta \\
& =\frac{1}{\alpha \xi}\left\{\int_{\alpha}^{\alpha+\xi} f(\tau) d \tau-\int_{0}^{\xi} f(\tau) d \tau\right\} \\
& \rightarrow \frac{1}{\alpha}[f(\alpha)-f(0)] \\
& \text { as } \xi \rightarrow 0 \text {. }
\end{aligned}
$$

Thus (6) and (8) give the existence of 


$$
\lim _{\xi \rightarrow 0} \frac{1}{\xi}[f(\xi)-f(0)]=\left\{\frac{1}{\alpha} \int_{0}^{\alpha} G_{1}[f(0), f(\zeta)] d \zeta\right\}^{-1} \frac{1}{\alpha}[f(\alpha)-f(0)] .
$$

Thus $f(\xi)$ is differentiable at $\xi=0$. Applying the lemma once more we have

$$
\begin{aligned}
\frac{1}{\eta}[f(\xi+\eta)-f(\xi)] & =\frac{1}{\eta}\{G[f(\eta), f(\xi)]-G[f(0), f(\xi)]\} \\
& =\frac{1}{\eta}[f(\eta)-f(0)] Q[f(\eta), f(0), f(\xi)] \\
& \rightarrow f^{\prime}(0) G_{1}[f(0), f(\xi)]
\end{aligned}
$$

uniformly for $0 \leqq \xi \leqq \omega$. The existence of the higher derivatives is readily established. We shall indicate the argument for the case of the second derivative. We have

$$
f^{\prime}(\xi)=f^{\prime}(0) \frac{1}{(2 \pi i)^{2}} \int_{\Gamma} \int_{\Gamma} G_{1}[\alpha, \beta] R[\alpha, f(0)] R[\beta, f(\xi)] d \alpha d \beta .
$$

It is readily shown that uniformly for $\beta$ on $\Gamma$ we have as $\eta \rightarrow 0$

$$
\frac{1}{\eta}\{R[\beta, f(\xi+\eta)]-R[\beta, f(\xi)]\} \rightarrow-f^{\prime}(\xi)\{R[\beta, f(\xi)]\}^{2} .
$$

Hence

$$
f^{\prime \prime}(\xi)=-f^{\prime}(0) f^{\prime}(\xi) \frac{1}{(2 \pi i)^{2}} \int_{\Gamma} \int_{\Gamma} G_{1}(\alpha, \beta) R[\alpha, f(0)] R[\beta, f(\xi)]^{2} d \alpha d \beta
$$

and a contour integral argument of familiar type shows that this expression equals $f^{\prime}(0) f^{\prime}(\xi) G_{11}[f(0), f(\xi)]$ where $G_{11}[\alpha, \beta]=\left(\partial^{2} / \partial \alpha \partial \beta\right) G[\alpha, \beta]$ and $G_{11}(u, v)$ is defined by the usual contour integral for $u, v \in B(\Delta)$. We see in particular that $f^{\prime \prime}(0)=\left[f^{\prime}(0)\right]^{2} G_{11}[f(0), f(0)]$ and hence is uniquely determined by $f(0)$ and $f^{\prime}(0)$. Similarly it may be shown that all higher derivatives exist and are uniquely determined by $f(0)$ and $f^{\prime}(0)$.

Now suppose that $g(\xi)$ is another continuous solution of equation (1) with $g(0)=f(0)$ and $g^{\prime}(0)=f^{\prime}(0)$. From the preceding remarks we see that $g^{(n)}(0)=f^{(n)}(0), n=1,2, \ldots$. From the contour integral representation of the function $Q[f(\xi), g(\xi), f(\eta)]$ combined with (5) we see that there is a finite $K=K(f, g)$ such that for $0 \leqq \xi, \eta \leqq \omega$

$$
|Q[f(\xi), g(\xi), f(\eta)]| \leqq K, \quad|Q[g(\eta), f(\eta), g(\xi)]| \leqq K .
$$

Since 


$$
G[f(\xi), f(\eta)]-G[g(\xi), f(\eta)]=Q[f(\xi), g(\xi), f(\eta)][f(\xi)-g(\xi)]
$$

we have

$$
|G[f(\xi), f(\eta)]-G[g(\xi), f(\eta)]| \leqq K|[f(\xi)-g(\xi)]|
$$

and a similar inequality in which $f, g$ and $\xi, \eta$ are interchanged. Placing $h(\xi)=f(\xi)-g(\xi)$ we have

$$
\begin{aligned}
h(\xi+\eta)= & f(\xi+\eta)-g(\xi+\eta) \\
= & G[f(\xi), f(\eta)]-G[g(\xi), g(\eta)] \\
= & G[f(\xi), f(\eta)]-G[g(\xi), f(\eta)] \\
& +G[g(\xi), f(\eta)]-G[g(\xi), g(\eta)] .
\end{aligned}
$$

Hence from (9) we have

$|h(\xi+\eta)| \leqq K\{|f(\xi)-g(\xi)|+|f(\eta)-g(\eta)|=K\{|h(\xi)|+|h(\eta)|\}$

whence

$$
|h(2 \xi)| \leqq 2 K|h(\xi)| .
$$

By repeated use of this inequality we get

$$
h(\xi) \leqq(2 K)^{m}\left|h\left(\xi 2^{-m}\right)\right|, \quad m=1,2,3, \cdots .
$$

Now consider

$$
g_{n}(\xi)=g(\xi)-\sum_{\nu=0}^{n} g^{(\nu)}(0) \frac{\xi^{\nu}}{\nu !}
$$

and let

$$
M_{n}[g]=\max \left|g_{n}^{(n)}(\xi)\right|=\max \left|g^{(n)}(\xi)-g^{(n)}(0)\right|
$$

in the interval $[0, \omega]$. Since

$$
g_{n}(\xi)=\int_{0}^{\xi} \cdots \int_{0}^{\xi_{n-2}} \int_{0}^{\xi_{n-1}} g_{n}^{(n)}\left(\xi_{n}\right) d \xi_{n} d \xi_{n-1} \cdots d \xi_{1},
$$

we have

$$
\left|g_{n}(\xi)\right| \leqq \frac{\xi^{n}}{n !} M_{n}[g]
$$

$0 \leqq \xi \leqq \omega$.

Since

$$
|h(\xi)|=|f(\xi)-g(\xi)|=\left|f_{n}(\xi)-g_{n}(\xi)\right| \leqq\left|f_{n}(\xi)\right|+\left|g_{n}(\xi)\right|,
$$

we see that $|h(\xi)| \leqq 2 \xi^{n} M_{n} / n$ !, where $M_{n}$ is the larger of $M_{n}[f]$ and 
$M_{n}[g]$. A combination of this inequality with (10) yields

$$
|h(\xi)| \leqq \frac{2}{n !} M_{n} \omega^{n}\left(2^{1-n} K\right)^{m}, \quad 0 \leqq \xi \leqq \omega, m, n=1,2, \cdots .
$$

Here we fix $n$ so that $2^{1-n} K<1$. Since $m$ is independent of $n$ and may be taken arbitrarily large we see that $h(\xi) \equiv 0$ and thus that $g(\xi)=f(\xi)$, $0 \leqq \xi \leqq \omega$.

Suppose now that $\phi(\xi)$ is a nonconstant analytic scalar solution of (1). Since $\phi$ is analytic at $\zeta=0$ we have $\phi(\zeta)=\sum \alpha_{n} \zeta^{n}|\zeta|<\rho$ and

$$
\phi\left(\zeta_{1}+\zeta_{2}\right)=G\left[\phi\left(\zeta_{1}\right), \phi\left(\zeta_{2}\right)\right], \quad\left|\zeta_{1}\right|,\left|\zeta_{2}\right|,\left|\zeta_{1}+\zeta_{2}\right|<\rho .
$$

Differentiating $\phi(\zeta)=G[\phi(\zeta), \phi(0)]$ we get $\phi^{\prime}(\zeta)=\phi^{\prime}(\zeta) G_{1}[\phi(\zeta), \phi(0)]$ and since $\phi^{\prime}(\zeta) \neq \equiv 0$ we have $G_{1}[\phi(\zeta), \phi(0)] \equiv 1$ and, in particular,

$$
G_{1}[\phi(0), \phi(0)]=1 \text {. }
$$

Differentiating (11) by parts with respect to $\zeta_{1}$ and putting $\zeta_{1}=0$ in the result one gets

$$
\phi^{\prime}(\xi)=\phi^{\prime}(0) G_{1}[\phi(0), \phi(\xi)]
$$

whence $\phi^{\prime}(0) \neq 0$. But if $\phi(\zeta)$ satisfies the conditions stated so does $\phi(\alpha \zeta)$ for any $\alpha \neq 0$. We can consequently normalize $\phi(\zeta)$ by assuming that $\phi^{\prime}(0)=1$. Suppose now that $f(\xi)$ is a continuous, and hence differentiable, solution of (1) such that $f(0)=\phi(0) e, f^{\prime}(0)=a$. Then $G_{1}[f(0), f(0)]=G_{1}[\phi(0), \phi(0)] e=e$ and hence it has an inverse. On the other hand the function $\phi(a \zeta)$ is given by the series $\sum \alpha_{n} a^{n} \zeta^{n}$ at least for $|\zeta|<\rho /|a|$ and for such values of $\zeta$ we have also $\sigma \phi(a \zeta)=\phi(\zeta \sigma a)$ $C \Delta$ since $|\sigma a| \leqq|a|$. From the construction of the series and the properties mentioned it follows that it satisfies (1) for $\left|\zeta_{1}\right|,\left|\zeta_{2}\right|$, $\left|\zeta_{1}+\zeta_{2}\right|<\rho /|a|$. Further, $\phi(0)=\phi(0) e=f(0), \phi^{\prime}(0)=a=f^{\prime}(0)$ and thus $f(\xi)=\phi(a \xi)$.

The Institute for Advanced Study and YALE UNIVERSITY 